\title{
Role of Cytokines in the Pathophysiology of Acute-on-Chronic Liver Failure
}

Qing Liu

Beijing You An Hospital, Beijing, China

\section{Key Words}

Liver injury $\cdot$ Cytokines $\cdot$ Hepatic progenitor cell

\begin{abstract}
Background: This is the first ever decade which has witnessed the emergence of a highly lethal condition termed acute-on-chronic liver failure (ACLF), which today is the leading cause of death compared to acute and chronic liver failure. However, the complex pathophysiology of ACLF remains poorly understood. This article will attempt to summarize recent progress in the understanding of the mechanisms of cytokines in ACLF. Methods: The search terms used on PubMed were cytokines, liver injury, complications of liver disease, and hepatic progenitor cell. Results: Cytokines play a significant role in the pathophysiology of ACLF including hepatocellular death, extrahepatic complications and hepatocyte regeneration. The tissue-damaging mechanisms of cytokines are closely related to their hepatocyte proliferation and regeneration function. Conclusions: It remains a challenge to selectively prevent the detrimental effects of cytokines. To design interventions which selectively target the detrimental effects of cytokines, a detailed understanding of cytokines in the pathophysiology ACLF is critical.

Copyright $\odot 2009$ S. Karger AG, Basel
\end{abstract}

\section{Introduction}

The natural course of chronic liver disease is often complicated by acute episodes of potentially reversible decompensation, triggered by a precipitating event such as infection or upper gastrointestinal bleeding, and is frequently referred to as acute-on-chronic liver failure (ACLF) [1, 2]. In a large-scale study [3] from 2000 to 2004 in China, of 799 patients with liver failure, $77.3 \%$ (618/799) were diagnosed as having ACLF and cirrhosis. Only 0.3 and $19 \%$ patients were diagnosed as having acute liver failure and subacute liver failure. Among patients with ACLF, 96.76\% (598/618) had HBV infection. The highest incidence of ACLF was found in subjects aged 46 years (range 12-80), suggesting that in the majority of patients, ACLF occurred during the third and fifth decades of life in the late phase in the natural history of chronic HBV infection. As the pool of patients with chronic liver disease grows, it is inevitable that a greater number of individuals with chronic $\mathrm{HBV}$ infection will be at risk for superimposed acute insult. Despite the advances in management and pharmacological therapy, patients very often deteriorate rapidly and progress to multiorgan failure.

In acute liver failure or ACLF, hepatocytes are exposed to high levels of a variety of cytokines, such as TNF- $\alpha$, IL-1, IFN- $\gamma$ and LPS [4-9]. These cytokines simultaneously activate both survival and apoptotic pathways and 
it depends on the level of pro- and antiapoptotic activities whether the balance will tip to one side or the other. Because the tissue-damaging mechanisms of cytokines are closely related to their hepatocyte proliferation and liver regeneration function, it remains a challenge to selectively prevent the detrimental effects. More recent data point to the concept of 'immune paralysis' in patients with ACLF, which is defined as a reduction in monocyte human leukocyte antigen-R (HLA-DR) expression and therefore an impairment in LPS-stimulated proinflammatory cytokine production (IL-1, IL-6, IL-8, TNF- $\alpha$ ) $[10,11]$. The correlation of immune paralysis with the severity of ACLF has not been well established. To design interventions, which selectively target the detrimental effects of cytokines, a detailed understanding of cytokines in the pathophysiology ACLF is critical. This article will attempt to summarize recent progress in the understanding of the mechanisms of cytokines in ACLF, including hepatocyte death, extrahepatic complications and hepatocyte regeneration.

\section{Role of Cytokines in Liver Injury}

Typical clinical findings of ACLF are: jaundice, coagulopathy and hepatic encephalopathy caused by massive/ submassive hepatocyte death. Hepatocyte death typically follows two patterns: necrosis and apoptosis [12]. Although necrotic cell death may also occur, hepatocyte apoptosis is apparently the major mechanism contributing to liver acute and chronic liver injury. The excessive and/or sustained apoptosis can lead to acute injuries, such as fulminant hepatitis and reperfusion damage, or even chronic sustained injuries, such as alcoholic liver disease, cholestatic liver disease, and viral hepatitis [13, 14]. Apoptosis occurs mainly via two signaling pathways: a death receptor-mediated extrinsic pathway or a mitochondria-mediated intrinsic pathway. Ligand/receptor binding induces the recruitment of several adapter proteins (Fas-associated protein with death domain (FADD), TNF-R1-associated death domain protein (TRADD), and proenzymes (i.e., procaspase- 8 and -10) at the intracellular domain of the receptor to form a complex usually referred to as DISC (death-inducing signaling complex)). The signal generated at the DISC by activated caspases results in cell death which, depending on the cell type, may or may not require the involvement of mitochondria for its execution. The intrinsic pathway is triggered by different extra- or intracellular signals, including ultraviolet and $\gamma$-irradiation, endoplasmic retic- ulum stress, growth factor deprivation, and oxidative stress with production of reactive oxygen species (ROS) trigger the intrinsic pathway via activation of proapoptotic members of the Bcl-2 family of protein (i.e., Bax, Bak), which oligomerize on the outer mitochondrial membrane and cause mitochondrial dysfunction. Following mitochondrial dysfunction, several apoptogenic factors are released from the mitochondrial intermembrane space into the cytosol, which contribute to protease activation and chromatin degradation (fig. 1) [15-17]. Oncotic necrosis and apoptosis can share features and mechanisms, which sometimes makes discrimination between two forms of cell death difficult. After ischemia/ reperfusion and other tissue stresses, cellular features of both apoptosis and necrosis often coexist. Pure apoptosis and pure necrotic cell death thus represent extremes in a continuous spectrum of cellular changes in dying cells. Recently, the term 'necrapoptosis' was introduced to emphasize that apoptosis and necrosis share common pathways and may not be so distinct as first proposed. Therefore, the mode of cell death can be changed from apoptosis to programmed necrosis and vice versa, which further supports the idea that necrosis is programmed and controllable [8].

Although very different agents can cause hepatocyte injury and liver failure, many studies in patients and animal models have strongly suggested that tumor necrosis factor (TNF)- $\alpha$ and Fas ligand (FasL) are involved in the induction of apoptosis and in triggering destruction of the liver, which ultimately leads to hepatic failure. Dysregulation of the TNF signaling pathway has been implicated in the pathogenesis of many chronic liver diseases, including viral hepatitis, alcoholic liver disease and fulminant liver failure, while altered TNF signaling and increased activation of the TNF-mediated apoptotic pathways are associated with steatohepatitis and other hepatocellular abnormalities [8-10].

An adherence-dependent neutrophil induced hepatocyte injury in a number of disease states, including hepatic ischemia-reperfusion, endotoxemia, sepsis, alcoholic hepatitis, remote organ injury, hemorrhagic shock, obstructive cholestasis, and certain drug-induced liver injuries [11]. The basic mechanism of a neutrophil-mediated pathophysiology in the liver requires the accumulation of primed and activated neutrophils in vascular beds of the liver, extravasation into the parenchyma, and the adherence-dependent cytotoxicity against hepatocytes. Neutrophils accumulate in the liver vasculature in response to the exposure to inflammatory mediators such as TNF- $\alpha$, IL-1 $\alpha$ or IL-1 $\beta$, CXC chemokines. Although 
Fig. 1. Hepatocyte death pathway (from Hengartner [84], with permission).

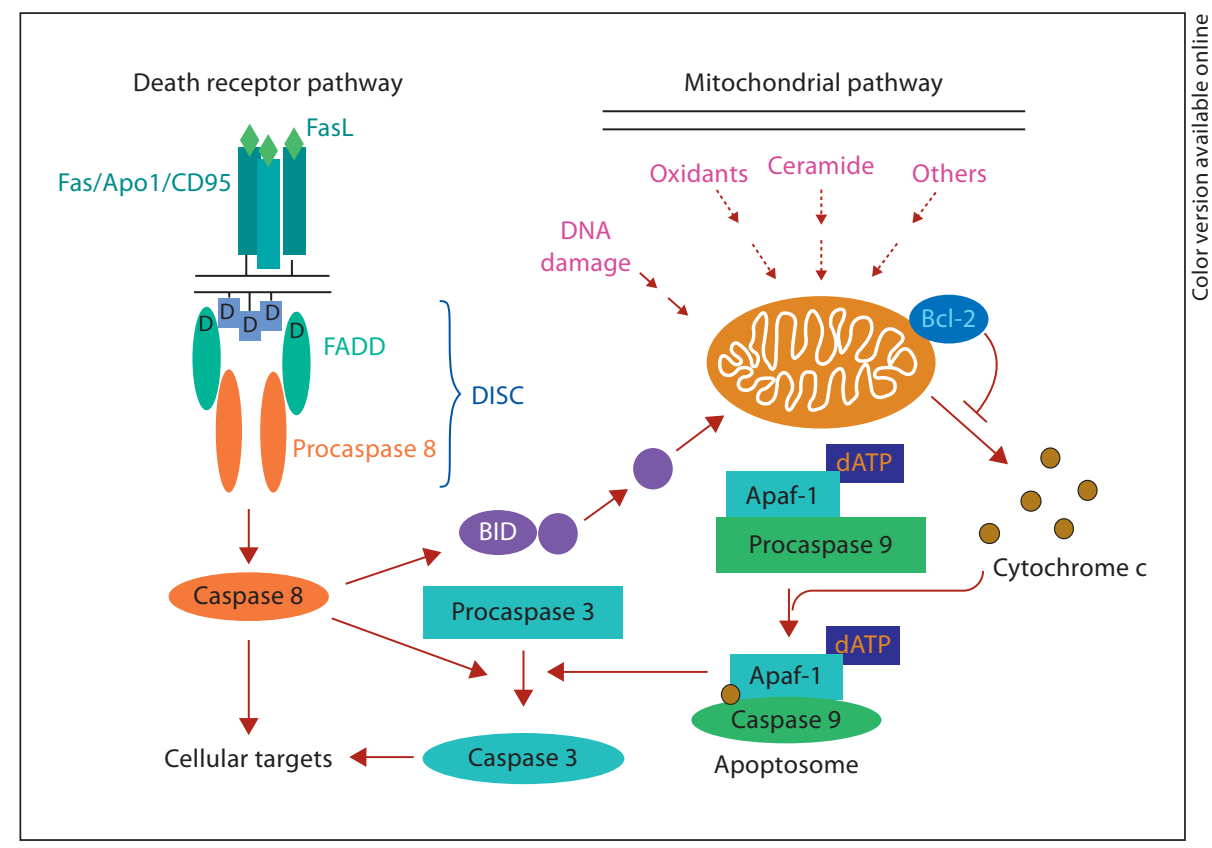

CXC chemokines are less potent activators of neutrophils than are cytokines and complement factors [12], excessive CXC chemokine formation in parenchymal cells can recruit neutrophils into the liver vasculature [13-15], induce transmigration, and cause injury [16]. The most convincing evidence for the importance of CXC chemokines under pathophysiological conditions was provided in hepatic ischemia-reperfusion injury $[17,18]$. Neutralizing CXC chemokines attenuated hepatic neutrophil recruitment and the neutrophil-mediated injury phase in rat and mouse models of warm ischemia-reperfusion $[19$, 20]. It was found that neutrophil extravasation and injury was independent of CXC chemokine formation in mice treated with Gal/ET and immunoneutralization of MIP2 and KC did not reduce LPS-induced leukocyte rolling and adhesion in post-sinusoidal venules [21]. Therefore, the role of CXC chemokines, i. e., macrophage inflammatory protein-2 (MIP-2) and cytokine-induced neutrophil chemoattractant $(\mathrm{KC})$, in leukocyte recruitment, microvascular perfusion failure, cellular injury, and apoptosis in the liver remains elusive.

\section{Role of Cytokines in Liver Complications}

The pathophysiological basis of ACLF is uncertain, but current hypotheses suggest that systemic inflammatory response may underlie the transition of a patient from a stable cirrhotic state to developing progressive liver injury and end-organ failure (fig. 2) [22].

Cirrhotic patients are particularly susceptible to bacterial infections because of increased bacterial translocation, possibly related to liver dysfunction and reduced reticuloendothelial function. Elevated serum levels of several cytokines, including soluble Fas (sFas) antigen [23], TNF- $\alpha$, sTNF- $\alpha$ R1, sTNF- $\alpha$ R2, IL-2, IL-2R, IL-6, IL-8, IL-10, and interferon- $\gamma$, have been described in patients with ACLF [24-28]. Elevated levels of circulating cytokines in ACLF may be the result of increased production due to endotoxemia $[29,30]$. A more recent study showed that presence of bacterial DNA in cirrhosis identifies a subgroup of patients with marked inflammatory response not related to endotoxin. Patients with bacterial DNA from Gram-positive microorganisms also showed significantly higher levels for both cytokines than patients without bacterial DNA, and similar to those in patients with bacterial DNA from Gram-negative bacteria $[25,26]$.

\section{Hyperdynamic Circulation}

Hyperdynamic circulation plays a central role in the development of the complications which is characterized by a high cardiac output, increased total blood volume, and a decreased splanchnic vascular resistance. In cirrhosis, portal hypertension defined as a portal pressure gradient of $\geq 12 \mathrm{~mm} \mathrm{Hg}$ could be the initiating factor of 
Fig. 2. Role of cytokines in liver complications.

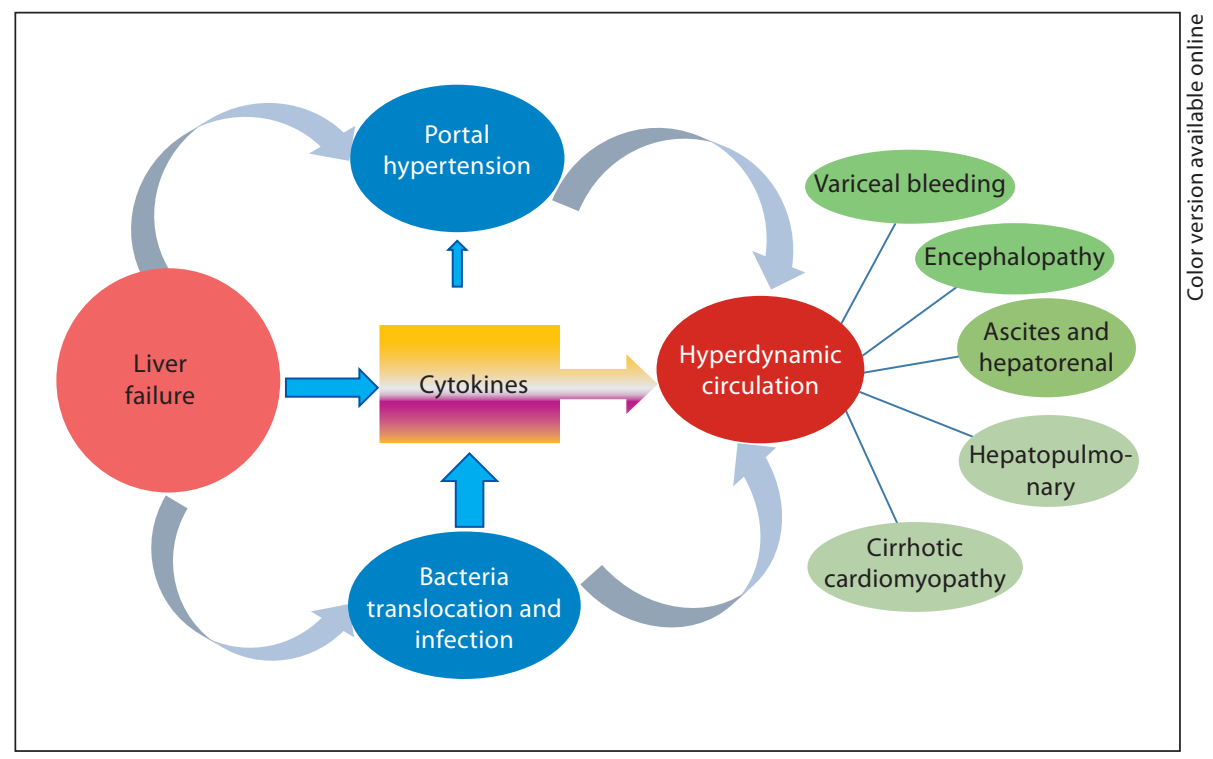

Table 1. The precipitating event as defined by the APASL Working Party on ACLF

\begin{tabular}{ll}
\hline Infectious etiology & Non-infectious etiology \\
\hline Hepatotropic and non-hepatotropic viruses & Alcohol: active drinking within 4 weeks \\
Reactivation of hepatitis B (overt or occult) or hepatitis C & Hepatotoxic drugs, herbs \\
Other infectious agents afflicting the liver & $\begin{array}{l}\text { Flare of autoimmune hepatitis or Wilson's disease } \\
\text { Variceal bleed }\end{array}$ \\
& Surgery \\
& Unknown hepatotoxic etiology \\
\hline
\end{tabular}

hyperdynamic circulation. A relatively new area of research in this field is the role of bacterial translocation from the gut in the initiation and maintenance of the hyperdynamic circulation syndrome [31, 32]. This relationship is supported by studies in patients with cirrhosis showing an amelioration of the hyperdynamic circulation through selective intestinal decontamination using poorly absorbed antibiotics [33].

ACLF was indentified as acute deterioration in liver function over a period of 2-4 weeks, usually associated with a precipitating event, in a patient with previously well-compensated chronic liver disease. The patients with ACLF showed hyperkinetic circulation with a low MAP $(69.7 \pm 1.1 \mathrm{~mm} \mathrm{Hg})$, an elevated cardiac index $\left(4.8 \pm 0.3 \mathrm{l} / \mathrm{min} / \mathrm{m}^{2}\right)$ and a low SVRI $(1,085 \pm 76 \mathrm{dyn} \cdot$ $\mathrm{s} / \mathrm{cm}^{5} / \mathrm{m}^{2}$ ) [34]. Whether a different mechanism of circulatory dysfunctions which occur in decompensated cirrhotics over a long period of time develop in ACLF in a shorter period is not fully understood.
A hyperdynamic circulation is also a common symptom in patients with systemic inflammatory response syndrome [35]. The development of systemic inflammatory response syndrome in patients with cirrhosis has been associated with the development of ACLF. In an inflammatory condition (an infectious or a non-infectious etiology, cf. table 1), with enhanced cytokines production, there are severe disturbances of the cardiovascular system - the circulation becomes hyperdynamic, cardiac output increases, and both blood pressure and systemic vascular resistance decrease. Persistent systemic hypotension associated with severe microcirculatory disturbance due to capillary leak and impaired oxygen extraction results in tissue hypoperfusion and hypoxia. If this cascade is not interrupted it will result in acute renal failure, cardiovascular and pulmonary insufficiency and, finally, multiple organ dysfunction [36]. 


\section{Hepatic Encephalopathy}

Hepatic encephalopathy (HE) is a neuropsychiatric syndrome associated with both acute and chronic liver dysfunction, and it has been theorized for over a century that neurotoxic substances are the cause. Patients may have altered brain energy metabolism and increased permeability of the blood-brain barrier. The latter may facilitate the passage of neurotoxins into the brain. Putative neurotoxins including short-chain fatty acids, false neurotransmitters (e.g., tyramine, octopamine, and $\beta$ phenylethanolamines), ammonia, and $\alpha$-aminobutyric acid (GABA) may gain entry into the brain. These neurotoxic substances may then contribute to morphologic changes in astrocytes. This may lead to increased intracranial pressure and, potentially, brain herniation [37, 38]. Therapy of HE is directed primarily at reducing ammonia generation and increasing its detoxification. One argument against the ammonia hypothesis is the observation that approximately $10 \%$ of patients with significant encephalopathy have normal serum ammonia levels [39]. Furthermore, many patients with cirrhosis have elevated ammonia levels without evidence for encephalopathy. Recent work demonstrated that HE is the interplay between ammonia, inflammatory responses and autoregulation of cerebral hemodynamics [40]. 84 such cirrhotics were studied by Briegel et al. [41]. The presence and severity of minimal HE were independent of severity of liver disease and ammonia concentration, but markers of inflammation were significantly higher in those with minimal HE compared with those without. The change in neuropsychological function following induced hyperammonemia is greater in those with more severe inflammation, suggesting that inflammation is an important determinant of the presence and severity of minimal HE. A significant positive correlation was found between serum levels of TNF and the severity of HE $(p<0.0001)$ $[42,43]$.

\section{Immune Paralysis}

Immune paralysis is defined as a reduction in monocyte human leukocyte antigen-DR (HLA-DR) expression and therefore an impairment in LPS-stimulated proinflammatory cytokine production (IL-1, IL-6, IL-8, TNF$\alpha)$. The correlation of immune paralysis with the severity of sepsis has been well established. A reduction in HLADR expression and ex vivo TNF- $\alpha$ production has been reported in ACLF patients and in CTP-C patients [4447].

As neutrophils play a crucial role in host defense against bacterial and fungal infections, neutrophil func- tions such as the production of ROS or phagocytosis have been studied in patients with ALF due to paracetamol overdose. Superoxide and hydrogen peroxide production by ALF neutrophils stimulated with zymosan opsonized with ALF serum was significantly reduced compared with the control subjects $(\mathrm{p}<0.01)$. Serum C3 complement levels were significantly reduced in ALF patients compared with control subjects $(p<0.0005)$ [48]. Abnormal neutrophil adherence has been described in patients with fulminant hepatic failure [49]. The mechanisms responsible for reduced neutrophil functions are not well understood, but it is believed that endotoxin renders them unable to respond to further bacterial challenge. This defective neutrophil function was transmissible through patients' plasma to normal neutrophils, and patients' neutrophil function could be restored by normal plasma [50].

An increase in killing correlated with increases in production of superoxide $(\mathrm{r}=0.96)$ and hydrogen peroxide $(\mathrm{r}=0.97)$ by ALF neutrophils after incubation with 1,000 and 5,000 IU/ml of G-CSF [51]. G-CSF administration is a safe and effective means of reversing the neutrophil defects of ALF, and may have a role in the prevention and treatment of infection in these patients. A dose of $50 \mu \mathrm{g} /$ $\mathrm{m}^{2} /$ day is as effective as higher doses and was associated with fewer side effects. Probiotic treatment would also restore neutrophil function in cirrhosis. A study showed that baseline neutrophil phagocytic capacity in patients was significantly lower compared to healthy controls (73 vs. $98 \%, \mathrm{p}<0.05)$, but normalized at the end of probiotic treatment $(\mathrm{n}=10,100 \%, \mathrm{p}<0.05)$. Soluble TNF receptor (sTNFR)-1 and -2 and IL-10 were significantly elevated and TLR4 expression normalized by the end of the study. No improvement was seen in disease controls [52]. The endotoxin removal strategies provide new targets for immune intervention.

\section{Cytokines in Regeneration and Repair of ACLF}

\section{Regeneration of ACLF Involved in Hepatic Progenitor Cell Activation}

Serial transplant studies in mice suggest that the hepatocyte possesses an essentially limitless regenerative capacity. However, there can be no survival after liver injury without regeneration $[53,54]$. As a general rule, replication of existing hepatocytes is the quickest and most efficient way to generate hepatocytes for liver regeneration and repair. Progenitor cells usually replicate and differentiate into hepatocytes only when the replication of 
Fig. 3. Progenitor cells, located within the smallest branches of the intrahepatic biliary tree, are a unique population, have a high nuclear to cytoplasmic ratio, and are activated in the face of liver injury. An extensive ductular reaction occurs after massive (or submassive) hepatic necrosis in humans, such as ACLF. In this type of injury, ductular proliferation involves mature cholangiocytes and ductular hepatocytes. The latter, located at the periphery of portal tracts, proliferate and express cholangiocyte and hepatocyte markers.

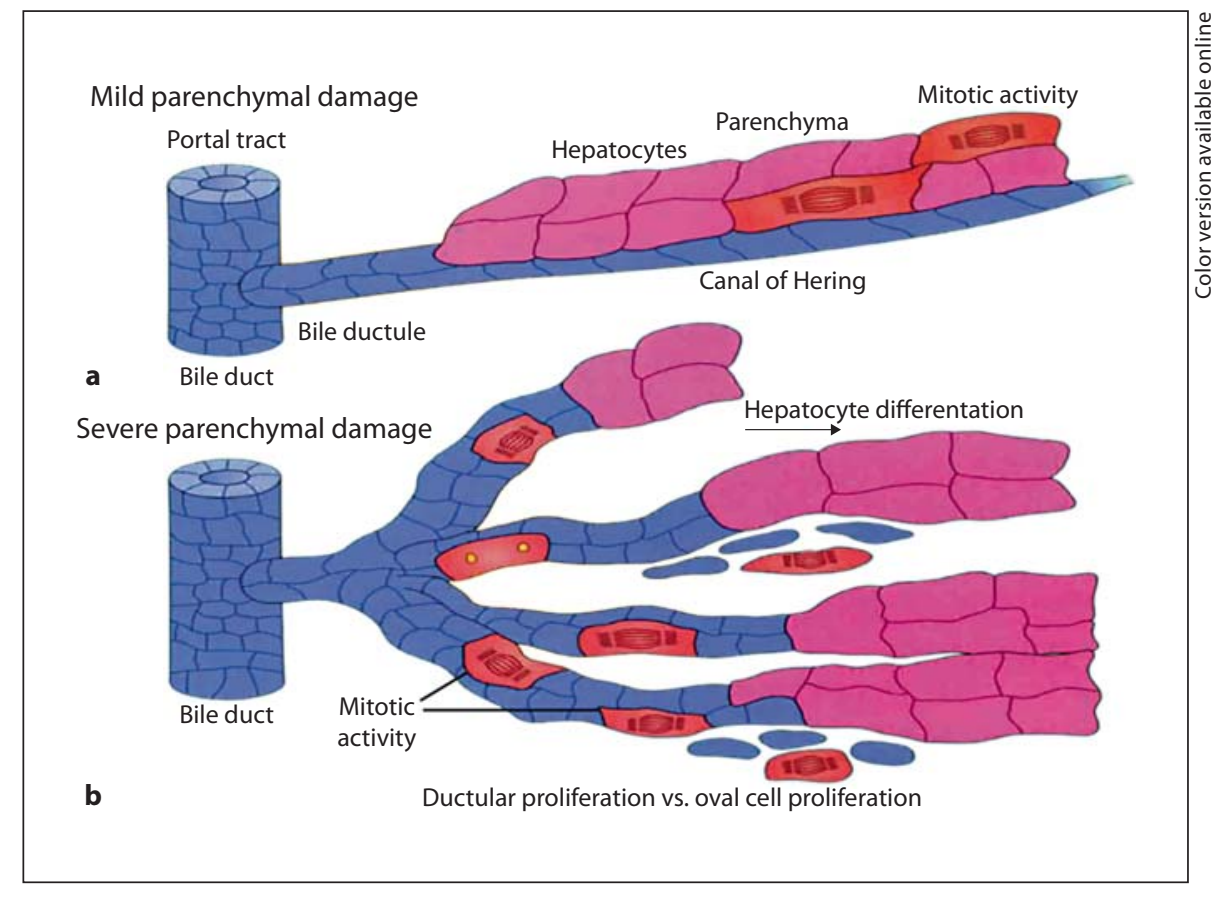

mature hepatocytes is delayed or entirely blocked. Bone marrow cells can generate hepatocytes in transplanted livers, but so far the frequency of hepatocytes produced by this route is very low, and such cells are not always detectable. ALCF is characterized by massive injury on the chronic preexisting liver diseases, thus the regeneration of ALCF may involve hepatic progenitor cell activation because (1) it is believed that a threshold of $50 \%$ loss of hepatocytes, associated with a significant decrease in the proliferative activity of remaining mature hepatocytes, is needed for extensive hepatic progenitor cell activation [55], and (2) impairment of liver regeneration is observed in chronic liver diseases, including non-alcoholic fatty liver disease, cirrhosis, chronic hepatic infection, malnutrition, with increased cell death, delayed mitosis and slower return of normal hepatic mass [56].

The progenitor cells, located within the smallest branches of the intrahepatic biliary trees, are a unique population, have a high nuclear to cytoplasmic ratio, and are activated in the face of liver injury. Progenitor cells express immature markers such as $\alpha$-fetoprotein, as well as mature hepatic markers (e.g., albumin) and biliary markers (e.g., cytokeratin-19) [57, 58]. An extensive ductular reaction occurs after massive (or submassive) hepatic necrosis in humans. In this type of injury, ductular proliferation involves mature cholangiocytes and ductular hepatocytes. The latter, located at the periphery of portal tracts, proliferate and express cholangiocyte and hepatocyte markers. Ductular hepatocytes are considered to be an intermediate form between ductular cells and hepatocytes (fig. 3) [59-63].

Katoonizadeh et al. [55] studied 74 patients with acute or subacute severe liver impairment by immunohistochemistry for CK7/CK19 (evaluation of progenitor cells activation/differentiation), Mib1(Ki-67)/P21 (evaluation of proliferative activity/proliferation arrest of hepatocytes) and hematoxylin and eosin (evaluation of hepatocyte loss). A positive correlation was found between the number of progenitor cells and clinical parameters of liver impairment such as the model for end-stage liver diseases.

It is unclear whether the generation of hepatocytes from progenitor cells leads to complete repopulation of injured human livers. Fujita et al. [64] performed sequential biopsies on the natural liver of a patient with massive necrosis after receiving an auxiliary partial orthotopic liver transplant. Complete regeneration of the natural liver was observed 12-14 months after transplantation, through a process that involved an initial ductular reaction followed by hepatocyte differentiation from progenitor cells. Nagasue et al. [65] evaluated remnant liver regeneration in normal liver and livers with chronic hepatitis and cirrhosis as well after major hepatic resection. A complete restoration of the residual liver size was found 
within 3 and 6 months in normal liver. In the patients with chronic hepatitis and liver cirrhosis, the remnant liver was enlarged but obviously more slowly. Among 4 patients with chronic hepatitis, only 1 had a rapid regeneration. Three patients with cirrhosis had a nearly normal liver volume at 9 and 12 months after operation. In 1 cirrhotic patient with extended right lobectomy, the residual liver has grown up to only one half of the normal size but he has been leading a normal life.

\section{Cytokines in Regeneration of Hepatic Progenitor Cells}

The process of liver regeneration requires a combination of cytokines and growth factors (table 2). Generally, EGF, TGF- $\alpha$ and HGF are considered as complete mitogens, i.e., each is capable of stimulating hepatocyte DNA synthesis in culture independently, co-mitogens such as insulin, glucagon, epinephrine, and norepinephrine potentiate the action of mitogens, but are unable to stimulate DNA synthesis alone $[66,67]$. It is clear that no one substance is singularly responsible for controlling this regeneration process. Also, it is puzzling that hepatocytes and intrahepatic progenitor cells (oval cells) have similar responses to most growth factors but rarely proliferate together.

HGF has attracted the greatest attention - it is synthesized by non-parenchymal liver cells and therefore affects hepatocytes in a paracrine manner. This was the first identified blood-borne hepatic mitogen in liver regeneration and remains a critical factor for liver growth along with its receptor c-Met. HGF is a potent inducer of DNA synthesis and regulates various processes in the liver [68]. Laboratory studies have shown that HGF plays a vital role in the complex orchestra of liver regeneration and that its mitogenic effect on the liver in part is through upregulation of another growth factor.

Stromal cell-derived factor-1 and its unique receptor CXCR4 are involved in the differentiation of progenitor cells. In severe liver injury combined with inhibition of mature hepatocyte proliferation such as ACLF, regeneration is achieved through the expansion and differentiation of liver precursor cells. Hepatic regeneration was induced by treating rats with 2-acetylaminofluorene, and followed by partial hepatectomy. Oval cells strongly expressed stromal cell-derived factor-1 protein and mRNA. CXCR4 mRNA hepatic level paralleled the number of oval cells and in situ hybridization showed CXCR4 mRNA expression by these cells. The results demonstrate that oval cells express SDF-1 as well as its receptor CXCR4 during hepatic regeneration from precursor cells, and suggest that the SDF-1/CXCR4 couple promotes the acti-
Table 2. Cytokines and other factors for liver regeneration

\begin{tabular}{ll}
\hline Factors & Cell cycle \\
\hline $\begin{array}{l}\text { Priming factors } \\
\text { TNF, IL-6 }\end{array}$ & G0 to G1 \\
$\begin{array}{l}\text { Growth factors } \\
\text { HGF, TGF- } \alpha \text {, EGF }\end{array}$ & G1 to S \\
$\begin{array}{l}\text { Co-mitogens } \\
\text { Insulin, epinephrine }\end{array}$ & Mitosis \\
\hline
\end{tabular}

vation of quiescent hepatic stem cells into oval cells and/ or stimulates oval cell proliferation through an autocrine/paracrine pathway. Stromal cell-derived factor- 1 is expressed in a broad range of tissues and is a potent chemoattractant for a variety of cells including hematopoietic stem cells, lymphocytes, and monocytes $[69,70]$.

As discussed, although liver regeneration typically occurs through replication of existing hepatocytes, oval cells proliferate only when hepatocyte proliferation is inhibited $[71,72]$. Transforming growth factor- $\beta$ (TGF- $\beta$ ) is a key inhibitory cytokine for hepatocytes, but not for oval cells. Both in vivo and in vitro, oval cells are less sensitive to TGF- $\alpha$-induced growth inhibition. TGF- $\beta$ levels are elevated when oval cells arise. It is an underlying mechanism for the proliferation of oval cells in an inhibitory environment such as chronic liver injury. Progenitor cell proliferation is associated with increased expression of c-kit, and also of hepatocyte growth factor, acidic fibroblast growth factor, and TGF, which also function as growth factors for hepatocyte replication. However, IFN- $\gamma$ increases in liver injury that involves only oval cell responses, but it is not upregulated during liver regeneration-involved hepatocyte proliferation such as partial hepatectomy. Studies in progress suggest that IFN- $\gamma$, in conjunction with TNF or LPS, can both inhibit hepatocyte proliferation through the generation of nitric oxide induced by nitric oxide synthase and stimulate oval cell replication.

TNF and IL- 6 are the key regulators of the initial steps of liver regeneration. TNF and IL- 6 can induce hepatocyte death on the one hand, it also promotes hepatocyte proliferation on the other hand, and both contribute to the priming pathways of restoration of liver mass after massive liver injury [73]. Current thinking is that one of the main roles of TNF- $\alpha$ is to regulate secretion of another cytokine, IL-6 [74]. The activation of NF- $\kappa$ B by TNF- $\alpha$ induces IL- 6 expression in Kupffer cells. Secreted IL-6 acts on neighboring hepatocytes in a paracrine fash- 


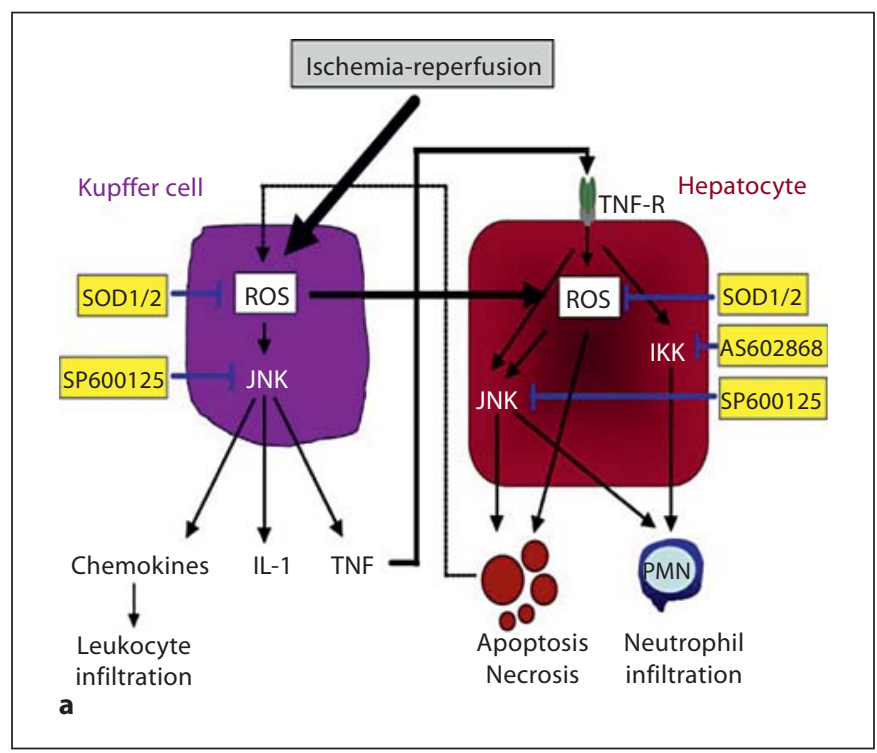

Fig. 4. Role of IKK, JNK, and ROS in liver injury and regeneration. a Kupffer cells generate ROS, which in turn activate JNK and enhance the secretion of various chemokines and cytokines including TNF- $\alpha$. TNF- $\alpha$ released from Kupffer cells may activate TNF receptors on hepatocytes to induce JNK and IKK activation as well as ROS production. b DEN-induced hepatocyte apoptosis

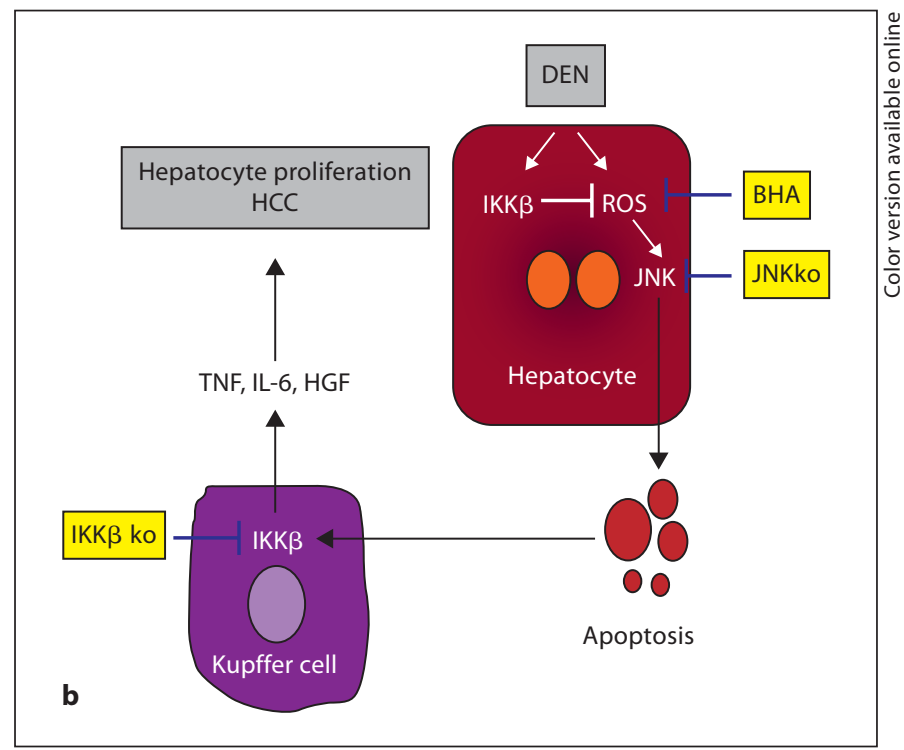

correlates with the activation of IKK in Kupffer cells, which in turn secrete mitogens such as IL-6, hepatocyte growth factor (HGF), and TNF- $\alpha$ to induce compensatory hepatocyte proliferation and hepatocarcinogenesis. Blocking IKK- $\beta$ in Kupffer cells reduces the secretion of mitogens and reduces DEN-induced hepatocarcinogenesis [61].

TNF- $\alpha$ may act as a potent activator of both proinflammatory and proapoptotic pathways, however the more recent finding from models of liver injury suggest that hepatocyte activation of NF- $\mathrm{\kappa B}$ plays a major role in the protection from cell death [79]. The cytoprotective role of TNF may involve two different mechanisms - direct and indirect.

Unlike Fas, cytokines like TNF- $\alpha$ are by themselves not sufficient to induce apoptosis. After TNF- $\alpha$ binding, these signaling pathways interact in a complex network at several levels, and activation of one pathway often depends on the inactivation of another one. Activation of TNF-R1 may lead to the activation of NF- $\kappa B$, JNK, and p38 through RIP1 and TRAF2, whereas activation of caspases and apoptosis is mediated through FADD [80]. Although death receptors of the TNF receptor family such as Fas and TNF-related apoptosis-inducing ligand (TRAIL) efficiently form a 'death-inducing signaling complex' (DISC) in which caspase- 8 activation is initiated, such a complex has not been detected in TNF receptor signaling. Most likely, TNF-R1 only induces a weak and transient formation of this complex because of the TRAF2-mediated recruitment of inhibitor of apoptosis (IAP) molecules, which interfere with the activation of 
caspase-8. This concept is further supported by the finding that TNF- $\alpha$-induced death signals generally require additional mitochondrial signals, whereas Fas is capable of inducing apoptosis independently of this mitochondrial pathway in many cell types.

Indirectly, TNF- $\alpha$ can also regulate hepatocyte proliferation by controlling the NF- $\mathrm{KB}$ transcription of mediators in Kupffer cells that drive hepatocyte proliferation. After hepatic I/R injury, TNF- $\alpha$, but not Fas, is a crucial mediator in hepatic reperfusion injury. The activation of IKK in Kupffer cells generates ROS, which in turn activates JNK and enhances the secretion of various chemokines and cytokines including TNF- $\alpha$ and IL- 6 . This role of IKK/NF- $\kappa$ B in hepatocytes is different from in Kupffer cells. In Kupffer cells, IKK- $\beta$ induces the transcription of proinflammatory and proproliferative mediators, whereas in hepatocytes, IKK- $\beta$ protects from TNF-induced apoptosis. This differential role of IKK/NF- $\mathrm{B}$ in hepatocytes and Kupffer cells was recently demonstrated in the diethylnitrosamine model of hepatocarcinogenesis (fig. 4): DEN induced ROS production, subsequent JNK activation, and hepatocyte apoptosis are increased in mice with a hepatocyte-specific deletion of IKK- $\beta$. This hepatocyte apoptosis correlates with the activation of IKK in Kupffer cells. The increase in hepatocyte death stimulated Kupffer cells to release proinflammatory and proproliferative mediators that enhance compensatory proliferation of surviving hepatocytes and hepatocarcinogenesis. The opposite results, decreased hepatocyte proliferation and hepatocarcinogenesis, were obtained when IKK- $\beta$ was deleted in Kupffer cells because of the diminished secretion of factors that are crucial in driving hepatocyte proliferation. Several points should be addressed from the above study: hepatocytes do not require direct activation of the NF- $\mathrm{B}$ pathway for proliferation and the role of IKK/NF- $\kappa B$ in hepatocytes and Kupffer cells were different. Not only are these results interesting in the study of cancer but also potential therapy for ACLF, for example in Kupffer cells we can specifically target IKK- $\beta$ to prevent hepatic inflammation and promote hepatocyte proliferation. We can also block JNK in both Kupffer cells and hepatocytes to prevent liver cells from dying, but it may also increase the chance of hepatocellular carcinoma [81-83].

We have tried to summarize the pathophysiological mechanisms of ACLF from very opposing thoughts, conflicting experimental and clinical data, and contradictory theories which have all led to major advances in our understanding of the role of cytokines and chemokines in liver injury and repair after ACLF diseases. In the future, as new hypotheses are generated, tested, and when found to be lacking, either modified or rejected, further progress must be made. Because effective therapy is based on sound biology, as we continue to gain a better understanding of the basic mechanisms involved in this syndrome, we undoubtedly will develop new and effective therapeutic strategies for the benefit of our patients [37].

\section{References}

1 Jalan R, Williams R: Acute-on-chronic liver failure: pathophysiological basis of therapeutic options. Blood Purif 2002;20:252261.

-2 Sen S, Williams R, Jalan R: Emerging indications for albumin dialysis. Am J Gastroenterol 2005; 100:468-475.

3 Jia JD, Zhuang H: A winning war against hepatitis B virus infection in China. Chin Med J 2007;120:2157-2158.

4 Odeh M, Sabo E, Srugo I, Oliven A: Relationship between tumor necrosis factor- $\alpha$ and ammonia in patients with hepatic encephalopathy due to chronic liver failure. Ann Med 2005;37:603-612.

5 Odeh M, Sabo E, Srugo I, Oliven A: Serum levels of tumor necrosis factor- $\alpha$ correlate with severity of hepatic encephalopathy due to chronic liver failure. Liver Int 2004;24: $110-116$.
6 6 Elsing C, Harenberg S, Stremmel W, Thomas $\mathrm{H}$ : Serum levels of soluble Fas, nitric oxide and cytokines in acute decompensated cirrhotic patients. World J Gastroenterol 2007; 13:421-425

7 Vanessa S, Peter K, Reingard A, et al: Effect of extracorporeal liver support by MARS and Prometheus on serum cytokines in acute-on-chronic liver failure. Crit Care 2006;10:R169.

$\checkmark 8$ Sen S, Davies NA, Mookerjee RP, et al: Pathophysiological effects of albumin dialysis in acute-on-chronic liver failure: a randomized controlled study. Liver Transpl 2004;10: 1109-1119.

9 Guo LM, Liu JY, Xu DZ, et al: Application of a molecular adsorbents recirculating system to remove $\mathrm{NO}$ and cytokines in severe liver failure patients with multiple organ dysfunction syndrome. Liver Int 2003;23(suppl 3):16-20.
10 Doughty L, Clark RS, Kaplan SS, et al: sFas and sFas ligand and pediatric sepsis-induced multiple organ failure syndrome. Pediatr Res 2002;52:922-927.

11 Ambrosino G, Naso A, Feltracco P, et al: Cytokines and liver failure: modification of TNF- $\alpha$ and IL- 6 in patients with acute-onchronic liver decompensation treated with Molecular Adsorbent Recycling System (MARS). Acta Biomed 2003;74(suppl 2):7-9.

12 Riordan SM, Williams R: Mechanisms of hepatocellular injury, multiorgan failure, and prognostic criteria in acute liver failure. Semin Liver Dis 2003;23:203-215.

$\checkmark 13$ Luedde T, Liedtke C, Manns MP, Trautwein $\mathrm{C}$ : Losing balance: cytokine signaling and cell death in the context of hepatocyte injury and hepatic failure. Eur Cytokine Netw 2002; 13:377-383. 
14 Guicciardi ME, Gores GJ: Apoptosis: a mechanism of acute and chronic liver injury. Gut 2005;54:1024-1033.

15 Ashkenazi A, Dixit VM: Death receptors: signaling and modulation. Science 1998;281: 1305-1308.

16 Green DR, Reed JC: Mitochondria and apop tosis. Science 1998;281:1309-1312.

17 Green DR, Kroemer G: The pathophysiology of mitochondrial cell death. Science 2004; 305:626-629.

18 Lemasters JJ: Necrapoptosis and the mitochondrial permeability transition: shared pathways to necrosis and apoptosis. Am J Physiol 1999;276:1-6.

-19 Schulze-Osthoff K, Ferrari D, Los M, et al: Apoptosis signaling by death receptors. Eur J Biochem 1998;254:439.

-20 Krammer PH: CD95 (APO-1/Fas)-mediated apoptosis: live and let die. Adv Immunol 1999;71:163.

-21 Galle PR, Krammer PH: CD95-induced apoptosis in human liver disease. Semin Liver Dis 1998;18:141.

-22 Bajt ML, Farhood A, Jaeschke H: Effects of CXC chemokines on neutrophil activation and sequestration in hepatic vasculature. Am J Physiol 2001;281:G1188-G1195.

-23 Faouzi S, Burckhardt BE, Hanson JC, et al: Anti-Fas induces hepatic chemokines and promotes inflammation by an NF- $\mathrm{B}$-independent, caspase-3-dependent pathway. J Biol Chem 2001;276:49077-49082.

24 Maher JJ, Scott MK, Saito JM, Burton MC: Adenovirus-mediated expression of cytokine-induced neutrophil chemoattractant in rat liver induces a neutrophilic hepatitis. Hepatology 1997;25:624-630.

25 Dorman RB, Gujral JS, Bajt ML, et al: Generation and functional significance of CXC chemokines for neutrophil-induced liver injury during endotoxemia. Am J Physiol 2005; 288:G880-G886.

-26 Okaya T, Lentsch AB: Cytokine cascades and the hepatic inflammatory response to ischemia and reperfusion. J Invest Surg 2003;16: 141-147.

-27 Gujral JS, Farhood A, Bajt ML, Jaeschke H: Neutrophils aggravate acute liver injury during obstructive cholestasis in bile duct-ligated mice. Hepatology 2003;38:355-363.

-28 Kato A, Gabay C, Okaya T, Lentsch AB: Specific role of interleukin-1 in hepatic neutrophil recruitment after ischemia/reperfusion. Am J Pathol 2002;161:1797-1803.

29 Lentsch AB, Yoshidome H, Cheadle WG, et al: Chemokine involvement in hepatic ischemia/reperfusion injury in mice: roles for macrophage inflammatory protein-2 and KC. Hepatology 1998;27:507-512.

30 Jaeschke H, Bajt ML: Critical role of CXC chemokines in endotoxemic liver injury in mice. J Leukoc Biol 2004;76:1089-1090.

-31 Iwasaki A, Medzhitov R: Toll-like receptor control of the adaptive immune responses. Nat Immunol 2004;5:987-995.
32 Medzhitov R: Toll-like receptors and innate immunity. Nat Rev Immunol 2001;1:135145.

33 Kawai T, Adachi O, Ogawa T: Unresponsiveness of MyD88-deficient mice to endotoxin. Immunity 1999;11:115-122.

34 Takeuchi O, Hoshino K, Akira S: Cutting edge: TLR2-deficient and MyD88-deficient mice are highly susceptible to Staphylococcus aureus infection. J Immunol 2000;165: 5392-5396.

35 Aliprantis AO, Yang RB, Weiss DS: The apoptotic signaling pathway activated by Toll-like receptor 2. EMBO J 2000;19:33253336.

36 Han KJ, Su X, Xu LG: Mechanisms of TRIFinduced interferon-stimulated response element and NF- $\kappa \mathrm{B}$ activation and apoptosis. J Biol Chem 2004;279:15652-15661.

37 Reichen J, Lebrec D: The future treatment of portal hypertension. Best Pract Res Clin Gastroenterol 2007;21:191-202.

38 Rodríguez-Vilarrupla A, Fernández M, Bosch J, Carles J: Current concepts on the pathophysiology of portal hypertension. Ann Hepatol 2007;6:28-36.

39 Garcia-Tsao G, Wiest R: Gut microflora in the pathogenesis of the complications of cirrhosis. Best Pract Res Clin Gastroenterol 2004;18:353-372.

40 Laleman W, Wilmer A, Evenepoel P, et al: Effect of the molecular adsorbent recirculating system and Prometheus devices on systemic haemodynamics and vasoactive agents in patients with acute-on-chronic alcoholic liver failure. Crit Care 2006;10:108.

41 Briegel J, Forst H, Haller M: Stress doses of hydrocortisone reverse hyperdynamic septic shock: a prospective, randomized, doubleblind, single-center study. Crit Care Med 1999;27:723-732.

42 Abraham E, Singer M: Mechanisms of sepsis-induced organ dysfunction. Crit Care Med 2007;35:2408-2416.

43 Sen S, Williams R, Jalan R: Acute-on-chronic liver failure: pathophysiological basis of therapeutic options. Liver Transplant 2004; 10:1109-1119.

44 Butterworth RF: Neuronal cell death in hepatic encephalopathy. Metab Brain Dis 2007; 22:309-320.

45 Butterworth RF: Pathogenesis of hepatic encephalopathy: new insights from neuroimaging and molecular studies. J Hepatol 2004; 39:279-285.

46 Wright G, Jalan R: Management of hepatic encephalopathy in patients with cirrhosis. Best Pract Res Clin Gastroenterol 2007;21: 110.

47 Shawcross DL, Wright G, Olde Damink SW, Jalan R: Role of ammonia and inflammation in minimal hepatic encephalopathy. Metab Brain Dis 2007;22:125-138.
48 Clapperton M, Rolando N, Sandoval L, et al: Neutrophil superoxide and hydrogen peroxide production in patients with acute liver failure. Eur J Clin Invest 1997;27:164-168.

49 Altin M, Rajkovic IA, Hughes RD, Williams $\mathrm{R}$ : Neutrophil adherence in chronic liver disease and fulminant hepatic failure. Gut 1983; 24:746-750.

50 Mookerjee RP, Stadlbauer V, Lidder S, et al: Neutrophil dysfunction in alcoholic hepatitis superimposed on cirrhosis is reversible and predicts the outcome. Hepatology 2007; 46:831-840.

51 Rolando N, Clapperton M, Wade J, et al: Granulocyte colony-stimulating factor improves function of neutrophils from patients with acute liver failure. Eur J Gastroenterol Hepatol 2000;12:1135-1140.

-52 Stadlbauer V, Mookerjee RP, Hodges S, et al: Effect of probiotic treatment on deranged neutrophil function and cytokine responses in patients with compensated alcoholic cirrhosis. J Hepatol 2008;48:945-951.

53 Murata H, Yagi T, Iwagaki H, et al: Mechanism of impaired regeneration of fatty liver in mouse partial hepatectomy model. J Gastroenterol Hepatol 2007;22:2173-2180.

54 Hines IN, Kremer M, Isayama F, et al: Impaired liver regeneration and increased oval cell numbers following T-cell-mediated hepatitis. Hepatology 2007;46:229-241.

55 Katoonizadeh A, Decaestecker J, Wilmer A, et al: The model for end-stage liver diseases score to predict outcome in adult patients with non-acetaminophen-induced acute liver failure. Liver Int 2007;27:329-334.

56 Rutherford A, Chung RT: Acute liver failure: mechanisms of hepatocyte injury and regeneration. Semin Liver Dis 2008;28:167-174.

57 Wang X, Foster M, Al-Dhalimy M, et al: The origin and liver repopulating capacity of murine oval cells. Proc Natl Acad Sci USA 2003; 100(suppl 1):11881-11888.

58 Wang J, Clark JB, Rhee GS: Proliferation and hepatic differentiation of adult-derived progenitor cells. Cells Tissues Organs 2003;173: 193-203.

59 Fausto N: Liver regeneration and repair: hepatocytes, progenitor cells, and stem cells. Hepatology 2004;39:1477-1487.

-60 Roskams T, Desmet V: Ductular reaction and its diagnostic significance. Semin Diagn Pathol 1998;15:259-269.

61 Kiss A, Schnur J, Szabo Z, Nagy P: Immunohistochemical analysis of atypical ductular reaction in the human liver, with special emphasis on the presence of growth factors and their receptors. Liver 2001;21:237-246.

62 Demetris AJ, Seaberg EC, Wennerberg A, et al: Ductular reaction after submassive necrosis in humans. Special emphasis on analysis of ductular hepatocytes. Am J Pathol 1996;149:439-448.

63 Haque S, Haruna Y, Saito K, et al: Identification of bipotential progenitor cells in human liver regeneration. Lab Invest 1996;75:699705. 
64 Fujita M, Furukawa H, Hattori M, et al: Sequential observation of liver cell regeneration after massive hepatic necrosis in auxiliary partial orthotopic liver transplantation. Mod Pathol 2000;13:152-157.

-65 Nagasue N, Yukaya H, Ogawa Y, et al: Human liver regeneration after major hepatic resection. Ann Surg 1987;206:30-39.

-66 Starzl TE, Watanabe K, Porter KA, Putnam CW: Effects of insulin, glucagon, and insulin/glucagon infusions on liver morphology and cell division after complete portacaval shunt in dogs. Lancet 1976;i:821-825.

-67 Michalopoulos GK: Liver regeneration. Cell Physiol 2007;213:286-300.

-68 Hu Z, Evarts RP, Fujio K, et al: Expression of transforming growth factor- $\alpha /$ epidermal growth factor receptor, hepatocyte growth factor/c-met and acidic fibroblast growth factor/fibroblast growth factor receptors during hepatocarcinogenesis. Carcinogenesis $1996 ; 17: 931-938$.

69 Mavier P, Martin N, Couchie D, et al: Expression of stromal cell-derived factor-1 and of its receptor CXCR4 in liver regeneration from oval cells in rat. Am J Pathol 2004;165: 1969-1977.

-70 Dalakas E, Newsome PN, Harrison DJ, Plevris JN: Hematopoietic stem cell trafficking in liver injury. FASEB J 2005;19:1225-1231.
71 Yeoh GC, Ernst M, Rose-John S, et al: Opposing roles of gp130-mediated STAT-3 and ERK-1/2 signaling in liver progenitor cell migration and proliferation. Hepatology 2007;45:486-494.

72 Campbell JS, Mitchell C, Yeoh GC, Fausto N: Differential regulation of rodent hepatocyte and oval cell proliferation by interferon- $\gamma$. Hepatology 2005;41:906-915.

73 Webber EM, Bruix J, Pierce RH, Fausto N: Tumor necrosis factor primes hepatocytes for DNA replication in the rat. Hepatology 1998;28:1226-1234.

74 Luedde T, Trautwein C: Intracellular survival pathways in the liver. Liver Int 2006:26: 1163-1174.

75 Hirano T, Nakajima K, Hibi M: Signaling mechanisms through gp130: a model of the cytokine system. Cytokine Growth Factor Rev 1997;8:241-252.

76 Cressman DE, Greenbaum LE, DeAngelis RA, et al: Liver failure and defective hepatocyte regeneration in interleukin-6-deficient mice. Science 1996;274:1379-1383.

$>77$ Tsamandas AC, Syrokosta I, Thomopoulos $\mathrm{K}$, et al: Potential role of hepatic progenitor cells expression in cases of chronic hepatitis $\mathrm{C}$ and their relation to response to therapy: clinicopathologic study. Liver Int 2006;26: 817-826.
78 Mullin EJ, Metcalfe MS, Maddern GJ: Artificial liver support potential to retard regeneration? Arch Surg 2004;139:670-677.

79 Schwabe RF, Brenner DA: Mechanisms of liver injury. I. TNF- $\gamma$ induced liver injury: role of IKK, JNK, and ROS pathways. Am J Physiol 2006;290:G583-G589.

80 Chaisson ML, Brooling JT, Ladiges W, et al: Hepatocyte-specific inhibition of NF- $\mathrm{B}$ leads to apoptosis after TNF treatment, but not after partial hepatectomy. J Clin Invest 2002;110:193-202.

-81 Schwabe RF, Bradham CA, Uehara T, et al: c-Jun-N-terminal kinase drives cyclin D1 expression and proliferation during liver regeneration. Hepatology 2003;37:824-832.

82 Chang L, Kamata H, Solinas G, et al: The E3 ubiquitin ligase itch couples JNK activation to TNF- $\gamma$-induced cell death by inducing cFLIP(L) turnover. Cell 2006;124:601-661.

83 Maeda S, Kamata H, Luo JL, et al: IKK couples hepatocyte death to cytokine-driven compensatory proliferation that promotes chemical hepatocarcinogenesis. Cell 2005; 121:977-990.

$>84$ Hengartner MO: The biochemistry of apoptosis. Nature 2000;407:770-776. 